\title{
THE SCHWARZIAN DISTANCE BETWEEN DOMAINS: A QUESTION OF O. LEHTO
}

\author{
BRAD OSGOOD and DENNIS STOWE
}

Möbius transformations are the only conformal mappings of $\overline{\boldsymbol{C}}$, and one common interpretation of the Schwarzian derivative $S(f)$ of an analytic function $f$ is that of measuring how close $f$ is to being a Möbius transformation. Thus $S(f) \equiv 0$ if and only if $f$ is Möbius, while if $f$ is locally injective in a quasidisk and has small enough Schwarzian then it will be injective and have a quasiconformal extension to $\bar{C}$, with dilatation controlled by the size of $S(f)$. By analogy, might it not also be the case that the size of the Schwarzian derivative of a conformal mapping between two domains defines a good measure of their distance apart, when domains which are conformally equivalent via a Möbius transformation are considered identical? We shall show that this cannot be pushed too far, answering in the negative a question asked by O. Lehto [L, p. 68]. We refer to Lehto's monograph for some basic definitions, results, and formulas we use here. We also note that the perspective leading to our particular construction comes very much from our work in [OS] on a generalization of the Schwarzian.

We consider simply connected domains $D \subset C$ with the Poincaré metric $\lambda_{D}|d z|$. If $f$ is a conformal mapping of $D$ and $S(f)=\left(f^{\prime \prime} \mid f^{\prime}\right)^{\prime}-\frac{1}{2}\left(f^{\prime \prime} \mid f^{\prime}\right)^{2}$ is its Schwarzian derivative then set

$$
\|S(f)\|_{D}=\sup \lambda_{D}^{-2}|S(f)| \text {. }
$$

Lehto defines a pseudometric on domains by

$$
\delta\left(D_{1}, D_{2}\right)=\inf _{f}\|S(f)\|_{D_{1}}
$$

where $f$ is a conformal mapping of $D_{1}$ onto $D_{2}$. Then $\delta$ is symmetric and satisfies the triangle inequality, and $\delta\left(D_{1}, D_{2}\right)=0$ if $D_{1}$ can be mapped onto $D_{2}$ by a Möbius transformation. Lehto asks if the converse of this last statement holds, that is, is $\delta$ a metric? We show that this is not the case.

Theorem 1. There exist domains $D_{1}$ and $D_{2}$ which are not Möbius equivalent, but for which $\delta\left(D_{1}, D_{2}\right)=0$. Moreover, the domains can each be chosen to be arbitrarily close to a disk (in terms of $\delta$ ).

Incidentally, it is true that if $D_{1}$ is a disk and if $\delta\left(D_{1}, D_{2}\right)=0$ then $D_{2}$ is a disk. 
Ultimately, we will exhibit the domains $D_{1}, D_{2}$ as images of the upper half-plane $\boldsymbol{H}$ under conformal mappings $f_{1}, f_{2}$. Then any conformal mapping $f$ of $D_{1}$ onto $D_{2}$ can be written in the form $f=f_{2} T f_{1}^{-1}$ where $T$ is a Möbius transformation of $\boldsymbol{H}$ onto itself. For $z \in D_{1}$ and $w=f_{1}^{-1}(z) \in \boldsymbol{H}$, the formula for the Schwarzian derivative of the composition of analytic functions, together with the conformal invariance of the Poincare metric, lead to

$$
\lambda_{D_{1}^{2}}^{-2}(z)|S(f)(z)|=\lambda_{H^{2}}^{-2}(w)\left|-S\left(f_{1}\right)(w)+T^{\prime}(w)^{2} S\left(f_{2}\right)(T w)\right| .
$$

This formula illustrates the role of $T$ in computing $\delta$; the functions $S\left(f_{1}\right)$ and $S\left(f_{2}\right)$ are fixed, and different choices for $T$ give different ways of shifting the latter before the former is subtracted.

The mapping functions $f_{1}, f_{2}$ will be obtained according to the following general scheme. Let $\varphi_{j}=\log \left|f_{j}^{\prime}\right|$. Then $f_{j}^{\prime \prime} \mid f_{j}^{\prime}=\left(\partial \varphi_{j} / \partial x\right)-i\left(\partial \varphi_{j} / \partial y\right)$, and $S\left(f_{j}\right)$ can be expressed in terms of $\varphi_{j}$ alone:

$$
S\left(f_{j}\right)=\left\{\frac{\partial^{2} \varphi_{j}}{\partial x^{2}}-\frac{1}{2}\left(\frac{\partial \varphi_{j}}{\partial x}\right)^{2}+\frac{1}{2}\left(\frac{\partial \varphi_{i}}{\partial y}\right)^{2}\right\}+i\left\{-\frac{\partial^{2} \varphi_{j}}{\partial x \partial y}+\frac{\partial \varphi_{j}}{\partial x} \frac{\partial \varphi_{j}}{\partial y}\right\} .
$$

To construct examples we start with a harmonic function $\varphi_{j}$ in $\boldsymbol{H}$, select an analytic function $h_{j}$ with real part $\varphi_{j}$, and let $f_{j}$ be an antiderivative of $e^{h_{j}}$. Without actually examining $f_{j}$ we can be sure that it is injective if, by bounding derivatives of $\varphi_{j}$, we obtain $\lambda_{H}^{-2}\left|S\left(f_{j}\right)\right| \leqq 2$. This follows from a Theorem of Nehari, [L, p. 90].

From a more geometric point of view, a harmonic function $\varphi_{j}$ on $\boldsymbol{H}$ gives a euclidean metric $e^{\varphi_{j}}|d z|$ on $\boldsymbol{H}$ and so admits a developing map $f_{j}: \boldsymbol{H} \rightarrow \boldsymbol{C}$. That is, $f_{j}$ is a conformal immersion with $\left|f_{j}^{\prime}\right|=e^{\varphi_{j}}$. Nehari's Theorem, as a condition on $\varphi_{j}$ via (2), gives a sufficient condition for this (euclidean) developing map to be an imbedding.

We continue with a few more general remarks. Let $p: \boldsymbol{R} \rightarrow \boldsymbol{R}$ be a real analytic function such that

$$
\left|p^{(k)}(t)\right| \leqq \varepsilon A^{k}, \quad t \in R, \quad k=0,1,2, \ldots
$$

for some positive constants $\varepsilon$ and $A$. It is convenient to write a point in $\boldsymbol{H}$ as $e^{t+i \theta}, t \in \boldsymbol{R}, \theta \in(0, \pi)$. Define

$$
\varphi\left(e^{t+i \theta}\right)=\Phi(p)\left(e^{t+i \theta}\right)=p(t)-\frac{p^{\prime \prime}(t)}{2 !}\left(\theta-\frac{\pi}{2}\right)^{2}+\frac{p^{(4)}(t)}{4 !}\left(\theta-\frac{\pi}{2}\right)^{4}-\ldots
$$

The series converges in the $C^{\infty}$ topology, and $\varphi$ is harmonic on $\boldsymbol{H}$. Let $g: H \rightarrow C$ be a developing map for the metric $e^{\varphi}|d z|$, i.e., $g$ is analytic with $\varphi=\log \left|g^{\prime}\right|$. From (2),

where

$$
S(g)\left(e^{t+i \theta}\right)=e^{-2 t}\{(u \cos 2 \theta+v \sin 2 \theta)+i(v \cos 2 \theta-u \sin 2 \theta)\}
$$

$$
\begin{aligned}
& u=\frac{\partial^{2} \varphi}{\partial t^{2}}-\frac{\partial \varphi}{\partial t}-\frac{1}{2}\left(\frac{\partial \varphi}{\partial t}\right)^{2}+\frac{1}{2}\left(\frac{\partial \varphi}{\partial \theta}\right)^{2} \\
& v=-\frac{\partial^{2} \varphi}{\partial t \partial \theta}+\frac{\partial \varphi}{\partial \theta}+\left(\frac{\partial \varphi}{\partial t}\right)\left(\frac{\partial \varphi}{\partial \theta}\right) .
\end{aligned}
$$


A rough estimate then gives

$$
|S(g)| \leqq e^{-2 t} \varepsilon C(\varepsilon, A), \quad C(\varepsilon, A)=\sqrt{2} A(1+A+\varepsilon A) \cosh ^{2}(A \pi / 2),
$$

and using $\lambda_{H}\left(e^{t+i \theta}\right)=\left(2 e^{t} \sin \theta\right)^{-1}$ we have that

$$
\left(\lambda \bar{H}^{2}|S(g)|\right)\left(e^{t+i \theta}\right) \leqq 4\left(\sin ^{2} \theta\right) \varepsilon C(\varepsilon, A) .
$$

In particular, a function constructed in this way will be injective if this bound is at most 2.

For our example we take $\varphi_{j}=\Phi\left(p_{j}\right), j=1,2$, where

$$
p_{1}(t)=\varepsilon \sum_{n=0}^{\infty} \frac{1}{2^{n+1}} \sin \left(\frac{t}{3^{n}}\right), \quad p_{2}(t)=-p_{1}(t) .
$$

Then $\left|p_{j}^{(k)}(t)\right| \leqq \varepsilon$ for all $k, t$. For $f_{j}, \log \left|f_{j}^{\prime}\right|=\varphi_{j}$, to be injective we have only to choose $\varepsilon$ so that $\varepsilon C(\varepsilon, 1) \leqq 1 / 2$. With $D_{j}=f_{j}(H)$ we see, actually, that we can make $\delta\left(D_{j}, H\right)$ arbitrarily small. In particular we can take $D_{1}$ and $D_{2}$ to be quasidisks [L, p. 88].

Let $s$ be a real number and let $T: \boldsymbol{H} \rightarrow \boldsymbol{H}$ be multiplication by $e^{s}$. Then $\left(T^{\prime}\right)^{2}\left(S\left(f_{2}\right) \circ T\right)=S\left(f_{2} \circ T\right)$, and $\log \left|\left(f_{2} \circ T\right)^{\prime}\right|=s+\varphi_{2} \circ T$. Now set $p(t)=-p_{1}(t)+$ $p_{2}(t+s)$ and let $\varphi=\Phi(p)$. As in (3) we find that

where

$$
\begin{gathered}
\left(-S\left(f_{1}\right)+\left(T^{\prime}\right)^{2}\left(S\left(f_{2}\right) \circ T\right)\right)\left(e^{t+i \theta}\right) \\
=e^{-2 t}\{(u \cos 2 \theta+v \sin 2 \theta)+i(v \cos 2 \theta-u \sin 2 \theta)\}
\end{gathered}
$$

$$
\begin{aligned}
& u=\frac{\partial^{2} \varphi}{\partial t^{2}}-\frac{\partial \varphi}{\partial t}-\frac{1}{2}\left(\frac{\partial \varphi}{\partial t}\right)\left(\frac{\partial \varphi_{1}}{\partial t}+\frac{\partial \varphi_{2}}{\partial t} \circ T\right)+\frac{1}{2}\left(\frac{\partial \varphi}{\partial \theta}\right)\left(\frac{\partial \varphi_{1}}{\partial \theta}+\frac{\partial \varphi_{2}}{\partial \theta} \circ T\right), \\
& v=-\frac{\partial^{2} \varphi}{\partial t \partial \theta}+\frac{\partial \varphi}{\partial \theta}+\frac{\partial \varphi}{\partial t} \frac{\partial \varphi_{1}}{\partial \theta}+\left(\frac{\partial \varphi_{2}}{\partial t} \circ T\right) \frac{\partial \varphi}{\partial \theta} .
\end{aligned}
$$

Let us take $s=3^{N} \pi$ where $N$ is a non-negative integer. Then

$$
\begin{aligned}
p(t) & =\varepsilon \sum_{n=0}^{\infty} \frac{1}{2^{n+1}}\left\{-\sin \left(\frac{t}{3^{n}}\right)-\sin \left(\frac{t+3^{N} \pi}{3^{n}}\right)\right\} \\
& =\varepsilon \sum_{n=N+1}^{\infty} \frac{1}{2^{n+1}}\left\{-\sin \left(\frac{t}{3^{n}}\right)-\sin \left(\frac{t+3^{N} \pi}{3^{n}}\right)\right\},
\end{aligned}
$$

whence

$$
\left|p^{(k)}(t)\right| \leqq \frac{\varepsilon}{2^{N}}, \quad k=0,1,2, \ldots
$$

Using these estimates in (5), we obtain

$$
\left|-S\left(f_{1}\right)+\left(T^{\prime}\right)^{2}\left(S\left(f_{2}\right) \circ T\right)\right| \leqq e^{-2 t} C(\varepsilon) / 2^{N},
$$


where $C(\varepsilon)=2 \sqrt{2} \varepsilon(1+\varepsilon) \cosh (\pi / 2)$. Thus for $f=f_{2} T f_{1}^{-1}$, a conformal mapping of $D_{1}$ onto $D_{2}$, we have

$$
\|S(f)\|_{D_{1}} \leqq 4 C(\varepsilon) / 2^{N} .
$$

Since $N$ is arbitrary, we conclude that $\delta\left(D_{1}, D_{2}\right)=0$.

It remains to show that $D_{1}$ and $D_{2}$ are not Möbius equivalent, which is to say that there is no Möbius transformation $T: \boldsymbol{H} \rightarrow \boldsymbol{H}$ such that

$$
-S\left(f_{1}\right)+\left(T^{\prime}\right)^{2}\left(S\left(f_{2}\right) \circ T\right) \equiv 0 .
$$

First note that on the imaginary axis we have from (3) that

$$
S\left(f_{1}\right)\left(e^{t+i \pi / 2}\right)=e^{-2 t}\left\{-p_{1}^{\prime \prime}(t)+p_{1}^{\prime}(t)+\frac{1}{2} p_{1}^{\prime}(t)^{2}\right\},
$$

a real number. If we take $t$ to be an even multiple of $\pi$ then

$$
\begin{aligned}
& p_{1}^{\prime}(t)=\varepsilon\left\{\frac{1}{2}+\sum_{n=1}^{\infty} \frac{1}{2^{n+1}} \frac{1}{3^{n}} \cos \left(\frac{t}{3^{n}}\right)\right\}, \\
& p_{1}^{\prime \prime}(t)=-\varepsilon \sum_{n=1}^{\infty} \frac{1}{2^{n+1}}\left(\frac{1}{3^{n}}\right)^{2} \sin \left(\frac{t}{3^{n}}\right) .
\end{aligned}
$$

Summing the geometric series then gives the estimates

$$
\begin{aligned}
\left|p_{1}^{\prime}(t)-\frac{\varepsilon}{2}\right| & \leqq \frac{\varepsilon}{10}, \\
\left|p_{1}^{\prime \prime}(t)\right| & \leqq \frac{\varepsilon}{34},
\end{aligned}
$$

so at the point $w=e^{t+i \pi / 2}$,

$$
\lambda_{H}^{2} S\left(f_{1}\right) \geqq-\frac{\varepsilon}{34}+\frac{\varepsilon}{2}-\frac{\varepsilon}{10}>\frac{\varepsilon}{3} .
$$

Next suppose that (6) holds for some Möbius transformation $T$. Again taking $w=e^{t+i \pi / 2}$ with $t$ an even multiple of $\pi$, we have

$$
\begin{aligned}
\lambda_{H}(T w)^{-2}\left|S\left(f_{2}\right)(T w)\right| & =\lambda_{H}(w)^{-2}\left|T^{\prime}(w)\right|^{2}\left|S\left(f_{2}\right)(T w)\right| \\
& =\lambda_{H}(w)^{-2}\left|S\left(f_{1}\right)(w)\right|>\frac{\varepsilon}{3} .
\end{aligned}
$$

On the other hand, (4) shows that $\lambda_{H}(T w)^{-2}\left|S\left(f_{2}\right)(T w)\right|$ can be made $<\varepsilon / 3$ if $\theta=\arg T w$ is near 0 or near $\pi$. It must be, therefore, that the image of the imaginary axis under $T$ is the imaginary axis, for it is a hyperbolic geodesic which, according to the above, is contained in some wedge $\eta<\theta<\pi-\eta$. Hence $T$ has one of the forms

$$
\begin{aligned}
& T w=e^{s} w, \\
& T w=-e^{s} / w
\end{aligned}
$$

for some $s \in \boldsymbol{R}$. 
We can discount these possibilities by examining equation (6) for (i) and (ii) along the imaginary axis. We obtain, respectively,

$$
\begin{aligned}
& -p_{1}^{\prime \prime}(t)+p_{1}^{\prime}(t)+\frac{1}{2} p_{1}^{\prime}(t)^{2}+p_{2}^{\prime \prime}(t+s)-p_{2}^{\prime}(t+s)-\frac{1}{2} p_{2}^{\prime}(t+s)^{2} \equiv 0, \\
& -p_{1}^{\prime \prime}(t)+p_{1}^{\prime}(t)+\frac{1}{2} p_{1}^{\prime}(t)^{2}+p_{2}^{\prime \prime}(s-t)-p_{2}^{\prime}(s-t)-\frac{1}{2} p_{2}^{\prime}(s-t)^{2} \equiv 0 .
\end{aligned}
$$

Consider (i). It is easy to show that for each $s$ there is a $t_{s}$ such that $p_{1}^{\prime}\left(t_{s}\right)=p_{2}^{\prime}\left(t_{s}+s\right)$. But then the functions $p_{1}^{\prime}(t)$ and $p_{2}^{\prime}(t+s)$ must be identical since they then solve the same initial value problem. However,

while

$$
p_{1}^{\prime}(0)=\varepsilon \sum_{n=0}^{\infty} \frac{1}{2^{n+1}} \frac{1}{3^{n}},
$$

$$
p_{2}^{\prime}(s)=-p_{1}^{\prime}(s)=-\varepsilon \sum_{n=0}^{\infty} \frac{1}{2^{n+1}} \frac{1}{3^{n}} \cos \left(\frac{s}{3^{n}}\right)<p_{1}^{\prime}(0),
$$

and we conclude that (i) cannot hold.

For (ii), replace $t$ by $s-t$, use $p_{2}=-p_{1}$, and subtract the resulting equation from (ii) to get

$$
p_{1}^{\prime}(t)^{2}=p_{2}^{\prime}(s-t)^{2}
$$

for all $t$. At $t=0$ we have $p_{1}^{\prime}(0)^{2}=p_{2}^{\prime}(s)^{2}$ which forces $s=0$. Substitute this information back into (ii), use $p_{2}=-p_{1}$ again, and the fact that $p_{1}^{\prime}$ is even while $p_{1}^{\prime \prime}$ is odd to get

$$
2 p_{1}^{\prime}(t) \equiv 0,
$$

an absurdity. Hence (ii) cannot hold, and we have shown that $D_{1}$ and $D_{2}$ are not Möbius equivalent.

We have answered Lehto's question, but one might now ask for a class of domains for which $\delta$ is a metric. Perhaps a different candidate for a metric should be proposed, ideally one that is complete. We do not know. We do, however, have one final (negative) comment. All of the properties and theorems we have used for the Schwarzian have direct analogues for the operator $f^{\prime \prime} \mid f^{\prime}$. One might then try to define a distance by

$$
\gamma\left(D_{1}, D_{2}\right)=\inf _{f}\left\|f^{\prime \prime} \mid f^{\prime}\right\|_{D_{1}}
$$

where $f: D_{1} \rightarrow D_{2}$ is a conformal mapping and $\left\|f^{\prime \prime}\left|f^{\prime} \|_{D_{1}}=\sup \lambda_{D}^{-1}\right| f^{\prime \prime}\left|f^{\prime}\right|\right.$. Here the relevant question is: if $\gamma\left(D_{1}, D_{2}\right)=0$, are $D_{1}$ and $D_{2}$ similar? Not surprisingly, the domains exhibited above show, with a few minor changes in the argument, that this is not so.

Theorem 2. There exist domains $D_{1}$ and $D_{2}$ which are not similar, but for which $\gamma\left(D_{1}, D_{2}\right)=0$. The domains can each be chosen to be arbitrarily close to a disk (in terms of $\gamma$ ). 


\section{References}

[L] Lehto, O.: Univalent functions and Teichmüller spaces. - Springer-Verlag, New YorkBerlin-Heidelberg-London-Paris-Tokyo, 1987.

[OS] OSGOoD, B., and D. STOWE: The Schwarzian derivative and conformal mapping of Riemannian manifolds. - Preprint.

Stanford University

Department of Mathematics

Stanford, California 94305

USA

Received 14 April 1987 